\title{
Determination of awareness, knowledge and behavior of a preclinical dentistry student group about the COVID-19 pandemic
}

\author{
(1) Neşe Oral, (1) Aslıhan Aslan Balcı, (1) Hilal Peker Öztürk, (1) İsmail Hakan Avsever \\ University of Health Sciences Turkey, Gülhane Faculty of Dentistry, Department of Dentomaxillofacial Radiology, Ankara, Turkey
}

Date submitted:

24.03.2021

Date accepted:

27.05.2021

Online publication date: 15.12.2021

\section{Corresponding Author:}

Neşe Oral, M.D., University of Health Sciences Turkey, Gülhane University Faculty of Dentistry, Department of Dentomaxillofacial Radiology, Ankara, Turkey

nese.oral@hotmail.com

ORCID:

orcid.org/0000-0003-2038-2897

Keywords: COVID-19, coronavirus, dental student, dentistry, pandemic

\begin{abstract}
Aims: Severe acute respiratory syndrome Coronavirus- 2 is usually transmitted by direct contact such as coughing and sneezing. In this survey, we aim to evaluate the knowledge of a group of preclinical dental students, who have not yet started working in the clinic, about the pandemic, protective measures, and the risks of infection in dental clinics.
\end{abstract}

Methods: In this descriptive study the criterion sampling method, one of the qualitative sampling techniques, was used to evaluate students with no clinical experience. The respondents were the preclinical dental students $\left(1^{\text {st }}, 2^{\text {nd }}\right.$ and $3^{\text {rd }}$ classes $)$. The survey questionnaire consisted of four domains: demographic information; general information about Coronavirus disease-2019 (COVID-19); preventive behaviors applied during the pandemic; and relationship of COVID-19 with dentistry.

Results: Of the students surveyed ( $n=95$, age between 18 and 23 years, female 67.4\%), 47 were from the $1^{\text {st }}$ class, 24 from the $2^{\text {nd }}$ class, and 24 from the $3^{\text {rd }}$ class. The least applied item among the preventive behaviors was the item "I have increased the frequency of cleaning the places that are in contact with the hands (e.g., door handle)" by $90.5 \%$. The majority of the responders $(97.9 \%)$ thought there was a high risk of transmission from the patient to the dentist. There were small differences in the direction of responses across student classes.

Conclusions: Dentistry students who participated in this study showed significant awareness of COVID-19, and were highly adapted to the preventive behaviors. However, the results also suggested some deficiencies in the knowledge level of students about the risk of viral transmission during dentistry procedures.

\section{Introduction}

A type of virus causing pneumonia in patients and rapidly spreading was detected in Wuhan, the Republic of China, in late December 2019 (1). On January 8, 2020, this virus was reported to be a coronavirus type by the Chinese Center for Disease Control and Prevention (2). The virus was called Severe acute respiratory syndrome Coronavirus-2 (SARS-CoV-2) by the International Committee on Taxonomy of Viruses (ICTV) (3). The disease caused by this virus, Coronavirus disease-2019 (COVID-19), was officially announced as an international emergency on January 30, 2020 and with the spread of this disease to dozens of countries, COVID-19 disease was declared by the World Health Organization (WHO) as a pandemic (4).
The first case in Turkey was diagnosed on 11th March 2020, andas of 23 December 2020 and 2.082.610 cases have been diagnosed with COVID-19 and 18.860 people have died due to coronavirus (5). The most important of the previous coronavirus types, including SARS and Middle East Respiratory syndrome outbreaks, had higher mortality rates, although they had a lower contagious rate compared to COVID-19 disease (6).

The most important symptoms of COVID-19 disease are dry cough, fever and shortness of breath (7). In addition, patients with a severe condition may develop complications such as pneumonia, pulmonary edema, Acute respiratory distress syndrome, multiple organ failure or death (8). WHO has informed the public that the duration of clinical symptoms to arise after contact with this virus is an average of 5 days and the incubation time is up to 14 days (9). 
The virus is usually transmitted by direct contact such as coughing and sneezing. Although the main source for transmission is symptomatic people, carrier individuals who are asymptomatic have also been reported to be effective in spreading this virus (10). It has been shown that SARS-CoV-2 virus can remain viable in aerosols emerging during dental procedures approximately for 3 hours. Therefore, dental clinics are considered among the places of a higher risk of cross infection for patients and the dental team $(11,12)$.

In order to prevent spread of the COVID-19 outbreak, it is important that the patients going to the dental clinics and the dentistry staff implement the protective measures recommended by the Ministry of Health in Turkey during the COVID-19 outbreak. In this survey study, we aimed to evaluate the knowledge of dentistry students, who have not yet started working in the clinics, about this pandemic, their protective measures, and the risks of cross infection in dental clinics.

\section{Methods}

\section{Study Design, Participants and Measures}

This was a descriptive, cross-sectional study. Criterion sampling method, which is one of the qualitative sampling techniques, was used to evaluate students with no clinical experience. This survey study was applied to volunteer preclinical dental students (1st, $2^{\text {nd }}$ and $3^{\text {rd }}$ classes) at the Department of Dentomaxillofacial Radiology, Gülhane University Faculty of Dentistry, University of Health Sciences Turkey, after the COVID-19 pandemic started. The questionnaire was prepared using the "Google Forms" application. In preparing the questions of the survey, a previous study on COVID-19 on Iranian Students was taken as a reference, and the questions adapted from the study by Taghrir et al. (13) (Table 1, 2). Some new questions about cross-infection in dentistry were also added to the survey (Table 3). Since it was a study conducted in Turkish society, the questions were prepared in Turkish.

In the first step, the pre-test of the prepared Google survey was conducted on approximately 10 people with similar characteristics to the target audience before starting this study. Then, the necessary corrections were made by considering the criticism and opinions of the participants in the pre-test questionnaire form, and the final form of the questionnaire was created after the duration of the questionnaire (about 5 minutes) was determined. The link of the form was sent through appliacations WhatsApp and Instagram to the students.

The questionnaire consisted of 4 main sections: demographic information such as gender, age, smoking; true/false questions for general information about COVID-19; questions about preventive behaviors applied during the COVID-19 pandemic; and questions about the relationship of COVID-19 disease with dentistry.

\section{Statistical Analysis}

Statistical analyses were performed using the Statistical Package for the Social Sciences (SPSS) software (version 22.0; SPSS Inc, Chicago, IL, USA) and Microsoft Excel 2016. Descriptive statistical methods and the criterion sampling method were employed.

The study was conducted in accordance with the Declaration of Helsinki. The questionnaire forms were delivered to the participants after approval by the Gülhane Scientific Research Ethics Committee of the University of Health Sciences Turkey, on 24.09.2020 (no. 2020-370). Informed consent was obtained from the participants.

\section{Results}

Of the students surveyed $(n=95$, age between 18 and 23 years, female $67.4 \%$ ), 47 were from the $1^{1 \text { st }}$ class, 24 from the $2^{\text {nd }}$ class, and 24 from the $3^{\text {rd }}$ class The youngest participant was 18 years old, the oldest participant was 23 years old, and the average age of the participants was 20.05 years. The majority of the students $(67.4 \%)$ were female $(n=64,67.4 \%)$. Sixteen $(16.8 \%)$ students were smoker. Smoking was more common among male participants ( $35.5 \%$ vs. $7.8 \%$ ).

Only 1 student answered "no" to the question "Do you know about the COVID-19 pandemic?". For the participants who answered "yes" to this question, another question was asked, "From which source did you get this information?". The answer options for this question were "International articles", "Media tools such as television, newspapers, magazines", "Social media", and "Other". The majority of the participants ( $76.8 \% / 73$ people) answered this question as "media tools such as television, newspapers and magazines" (Figure 1).

During the period when the questions were answered by the participants, only the persons diagnosed with the disease were recommended to wear a mask by the Ministry of Health, but as the pandemic progressed over time, it was recommended that everyone wore a mask. For this reason, since the answer to the item "All people should wear a mask" changed in time, this question was excluded from during the statistical analysis. This question was lilnked to the options of "true", "wrong", and "do not know". The average rate of correct answers given to the remaining 12 questions was $85.7 \%$. The two items with the lowest correct response rate were "N95 mask is required only during intubation, bronchoscopy and cardiopulmonary resuscitation $(\mathrm{T})(38.9 \%)$ " and "It can be treated with normal antiviral drugs (F) (54.7\%)". In addition, these 2 items included an "I don't know" option which was marked at the highest rate. Regarding the inter class comparisons, , the correct response rates were highest for the 3rd grade students, while the were lowest for the 1 st grade students $(84.72 \%>83.68 \%>80.14 \%)$ (Table 1). 


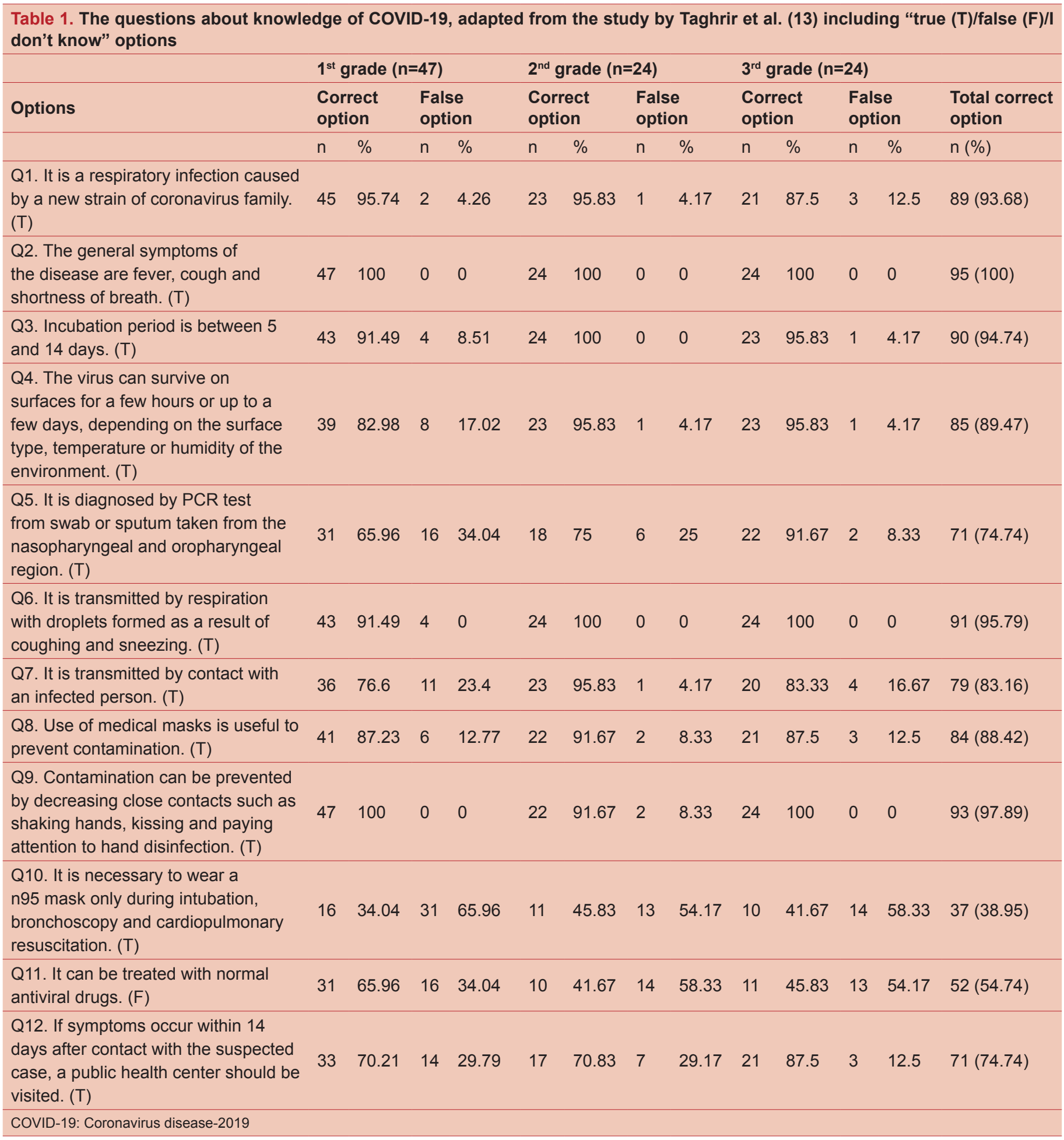

According to the answers to questions about preventive behavior, the proportion of people who performed these behaviors was 96\%. The least applied item among these preventive behaviors was "I have increased the frequency of cleaning the places that are in contact with the hands (door handle, etc.)" with a rate of $90.5 \%$.

The items in the risk perception section were "I argued with my family and friends about preventing COVID-19", "I can be infected more easily than people with COVID-19 disease", and "I am afraid of being infected with COVID-19 disease". The ratio of the students who positively responded to these items were $95.8 \%, 18.9 \%$, and $60 \%$. Responses to the preventive behaviors and perceptions are shown in Table 2.

Considering the responses to the questions about the spread of COVID-19 disease in dental clinics (Table 3), $97.9 \%$ of the students thought that dental clinics were environments 


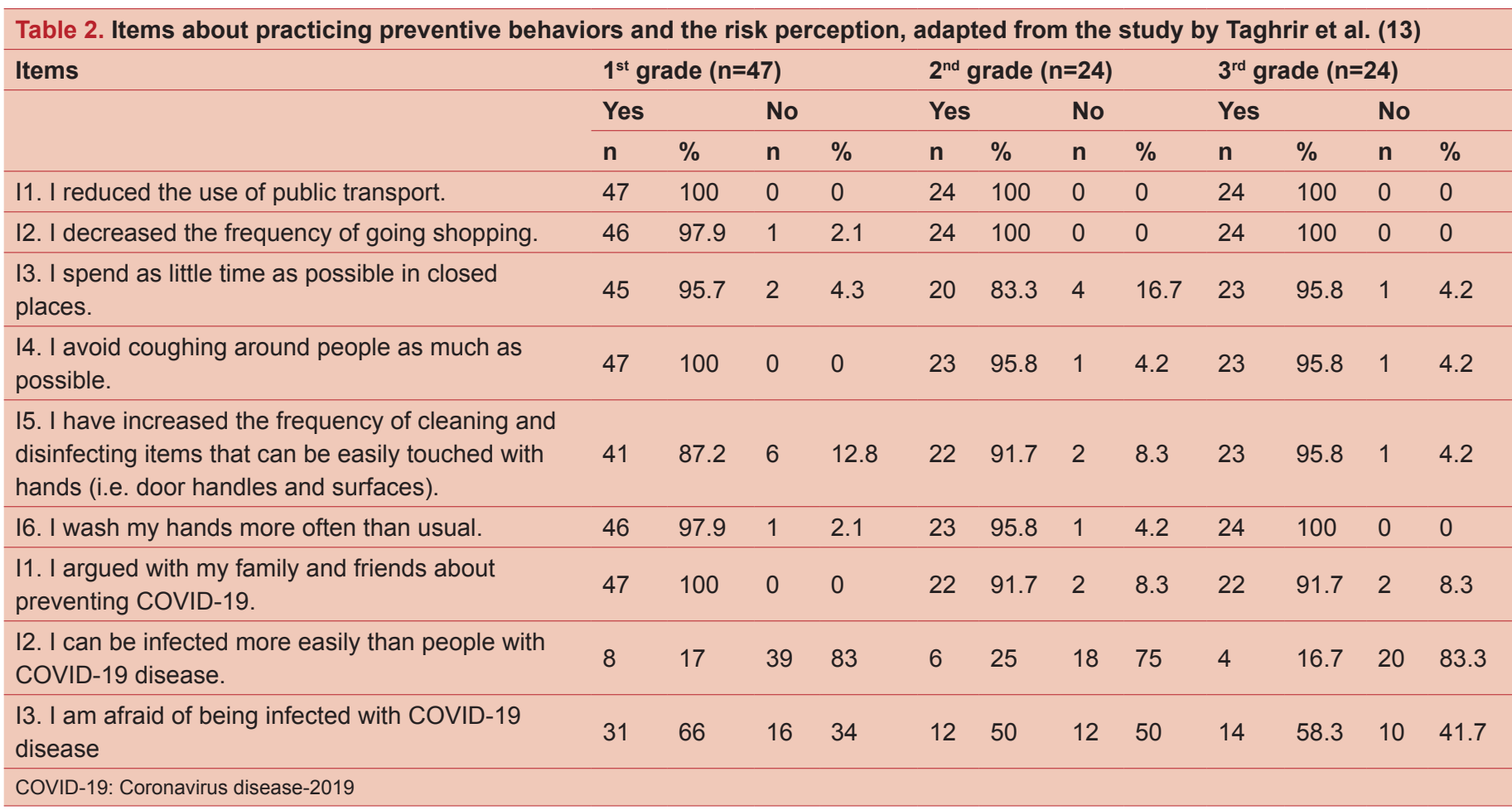

with a high risk of cross-infection between dentists and patients; $96.8 \%$ of the students thought that not to apply to dental clinics for procedures other than emergency treatments.

By the $87.4 \%$ of the participants high-speed hand tools used during dental treatment were thought to be effective in reducing the spread of the COVID-19 virus and $77.9 \%$ of the participants thought that standard preventive measures in dental clinics were adequately effective in the prevention of spread.

Of the participants, $81.1 \%$ positevly responded to the question "Does it pose a risk for the spread of the COVID-19 when the patient shows his aching tooth with his finger during the examination?". The number of students who answered "Yes" to the question "Is panoramic radiography or computed tomography a riskier imaging method than intraoral radiography in terms of the spread of the virus?" was only 5 , while the majority $(61.1 \%)$ chose the answer "I don't know",. For the question "Is the use of high volume saliva absorbers effective in reducing the production of droplets and aerosols?", $36.8 \%$ of the participants chose the "I don't know" option, while $44.2 \%$ of the students answered "Yes" to this question,.

Regarding the answers to the multiple choice questions related to the procedures within the scope of emergency dental treatments, only 13 students marked the options ("Shortterm tooth sensitivity", "Severe toothache caused by pulpal inflammation", "Tooth abscess" and "Trauma-related dental avulsion/luxation"). Responses to the options of emergency dental treatments are shown in Figure 2.

Of the students, $84.4 \%$ stated that the dentist who performed emergency dental treatment during the COVID-19 pandemic

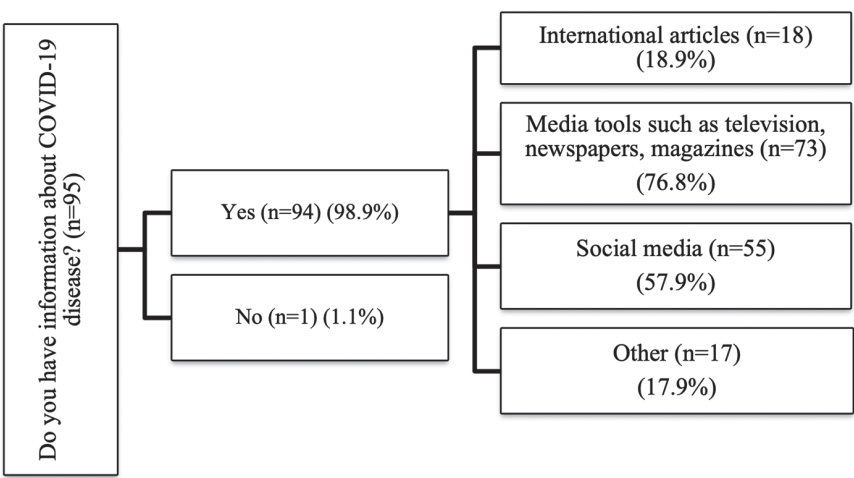

Figure 1. Information source about Coronavirus disease-2019

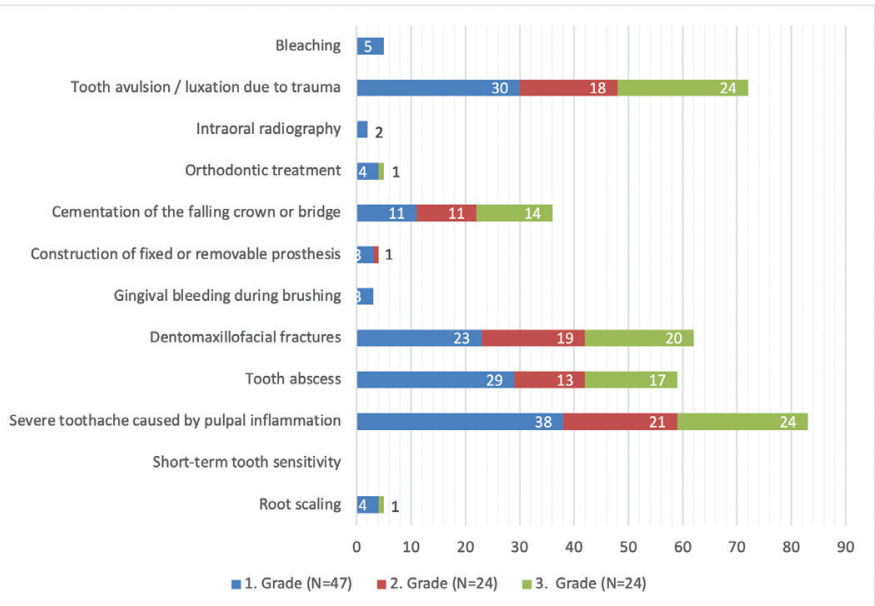

Figure 2. Emergency dental procedures 


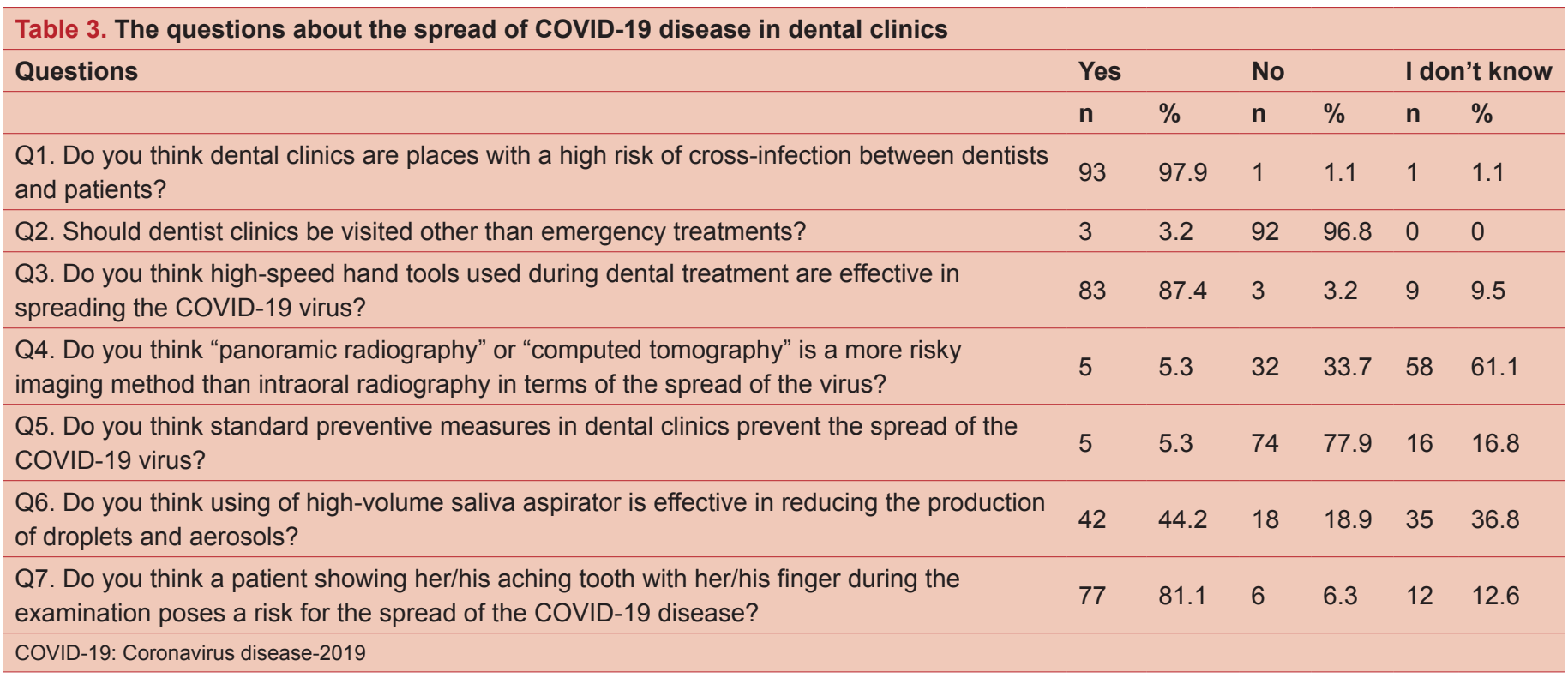

should use "Gloves, glasses, mouth mask, face mask, sterile disposable dress" together. For the question "Which one or ones are sufficient for the patient who will apply to the dental clinic during the COVID-19 outbreak?", 24\% of the students chose the answer of "glove and mouth mask". The rate of students who marked only the "mouth mask" option was $15.6 \%$, and the rate of students who marked all options (Gloves, glasses, mouth masks, face masks, sterile disposable clothes) was $15.6 \%$.

For the question "Which one or ones would be appropriate when the patient returns home after dental treatment?", 98.9\% of the participants thought that their hands should be washed. The rate of those who marked "Should wash my hands, put my clothes into dirty, take a shower" options together was $86.5 \%$.

\section{Discussion}

Healthcare workers and students are always at higher risk than other people concerning the infectious diseases. In order for health policy makers to make appropriate planning, it is important to evaluate knowledge of preventive behaviors and to understand the risk perception and anxiety level of health students.

The protocol of this survey had to be modified in a single item during the collection of data, indicating the dynamic changes and updates during the worst pandemic of the last centruy. The item that "All people should wear masks", which is one of the 13 items containing general information about COVID-19, was not included in the survey results because, in the period when the surveys were answered (March-April 2020), health managers stated that it would be sufficient for people diagnosed with COVID-19 to wear a mask, while it was stated that everyone should wear a mask during the period when the statistics of the survey results were made. The majority of the students correctly answered the questions with "true/false/do not know" options containing general information about COVID-19, in similar to a study previously which was conducted on medical students and reported this rate as $86.96 \%$ (13). In this context, the 2 items with the lowest rate should cautiously be evaluated. Less than half of the students answered the item "It is necessary to wear an N95 mask only during intubation, bronchoscopy and cardiopulmonary resuscitation" and slightlu more than half of the students correctly answered the item "COVID-19 disease can be treated with normal antiviral drugs". More training about the N95 and other similar standard masks and drugs should be provided to $1^{\text {st }}, 2^{\text {nd }}$ and $3^{\text {rd }}$ gradestudents of the dentistry faculty, who start clinical internships and treat patients soon. This could be the primary measure to protect them form infectious diseases both during their internships and their professional life.

When the classes are compared, the correct response rates increased, as we expected, from the first grade to the third grade students $(80.14 \%<83.68 \%<84.72 \%)$. However, although there were slight differences between the rates, they are very close to each other. The reason for this can be explained by the fact that none of the students had clinical experience when surveyed.

In a similar study conducted on healthcare professionals (doctors, nurses, and pharmacists) in Pakistan, $88.7 \%$ of the participants stated that they performed preventive measures during the COVID-19 period. On the other hand, this rate was higher in our study, almost all the respondents chose to perform preventive behaviors (14). The item with the lowest rate of response was the item "I have increased the frequency of cleaning the places that are in contact with hands (door handle, etc.)", though still with a rate of $90.53 \%$. The outcome to be drawn from this finding is that students should be given more information about frequently contacted areas and surface disinfection. 
Around one-fifth of the students thought that they would be more easily infected with the COVID-19 virus than other people. As we expected, the students in our study did not place themselves in a different stiuation from the other people because they had not yet treated patients and these results were lower compared to the similar previous two studies. In the survey study conducted by Ataş and Talo Yildirim (15) on dentistry students, $77.6 \%$ of preclinical students stated that they were afraid of being infected with COVID-19 disease. In another survey (16) on dentists, $54.5 \%$ of the participants strongly agreed that there was a high risk of contracting the COVID-19 in dental practice.

Aerosols that occur during dental treatments are considered a risk factor for cross-infection in the dental clinics, due to longterm dental treatments and close contact between the dentist and patient. In addition, the aerosols that occur during dental treatments pose a risk for the other people in the dental clinic because of their suspension in the air for a long time (17). In a previous survey study conducted on the parents of children (aged: 0-14) who visited the dental department, $91.89 \%$ of the participants stated that the virus could be transmitted to their children during an intervention (18). The result in our study was slightly higher, $97.9 \%$ of the participants thought that dental clinics were environments with a high risk of cross-infection.

Acute infections in the oral cavity, severe toothaches and traumas affecting the jaw require urgent intervention. Accordingly, it is recommended to dental clinics by healthcare professionals not to apply for aesthetic dental treatments and painless dental caries treatments during the COVID-19 pandemic. In accordance with these recommendations, a vast majority of the students thought that people should not apply to the dental clinic during the pandemic period. According to the report of the Republic of Turkey Ministry of Health, the procedures are considered as emergency dental treatment. The relevant emergency treatment limitations adapted from the study by Falahchai et al. (19) and the study by Oral et al. (20) are as follows:

a. Severe toothache caused by pulpal inflammation

b. Severe pain caused by pericoronitis or third molar

c. Postoperative osteitis or alveolitis

d. Abscess or bacterial infection that causing localized pain and swelling

e. Tooth fracture causing pain or soft tissue trauma

f. Tooth avulsion/luxation caused by trauma

g. Dentomaxillafacial traumas

h. Pain and/or infection due to injury in soft tissue as a result of breakage of brackets and wires of patients undergoing orthodontic treatment

i. Feeding plate applications of patients with newborn cleft plate

j. Temporamandibular joint k. Biopsy (in cases of suspected malignancy) (19)

I. Acute and painful lesions/ulcerations of the oral mucosa,

$\mathrm{m}$. Life-threatening or uncontrolled bleeding

n. Intraoral/extraoral infections that threaten the patient's airway patency

o. Treatment of patients who are undergoing radiotherapy and/or organ transplants

p. Dental consultation for medical problems

q. Taking sutures

r. Treatment so as not to form an aerosol for temporary restoration loss/fractures injuries preventing the use of removable dentures (20).

In the current study, four urgent procedures were given as the right choice for the 12-choice questions in which emergency procedures in dental practices were questioned; "Severe toothache caused by pulpal inflammation, tooth abscess, dentomaxillafacial traumas, tooth avulsion/luxation caused by trauma". Less than one-fifth of the participants in the survey correctly answered the emergency treatments in dentistry. As expected, the highest scores were "Severe toothache caused by pulpal inflammation",,"Tooth avulsion/luxation caused by trauma", "Dentomaxillafacial traumas" and "Tooth abscess", with responses higher than $50 \%$. In a study performed by Oral et al. (20) $17.4 \%$ of the participants answered the options of "severe toothache, tooth abscess, jaw and facial fractures and tooth dislocation as a result of trauma" for a similar question, by correctly marking their options.

Close contact of dentists and nurses with the patient's oral area during dental treatments, aerosols and saliva scattered into the air with high-speed hand tools used during dental treatments increase the risk of the spread of the virus. The use of high-volume saliva absorbers reduces the spread of the virus by reducing the scattering of droplets and aerosols into the air (21). Similarly, up to $90 \%$ of the students in our study thought that high-speed hand tools used during dental treatments were effective in the spread of COVID-19 virus and a low percentage of participants thought that high-volume saliva absorbers used during dental treatments were useful to reduce the droplets and aerosols. Therefore, to prevent the spread of COVID-19 virus and other droplet-borne microorganisms, the frequency of use of high-speed hand tools should be reduced and high-volume saliva absorbers should be used during dental treatments.

The majority of the students stated that the dentists who performed emergency dental treatment during the COVID-19 epidemic chose the option to use "gloves, glasses, mouth masks, face masks, sterile disposable clothes", and around onefifth of the students found it sufficient for patients admitted to the clinic to use "gloves and mouth masks". In a study conducted by 
Ahmed et al. (22) on patients admitted to a dental clinic, $60 \%$ of participants stated that the dentist and patient should wear "gloves, face masks and protective clothes".

This study has some limitations. Firstly, it was difficult to reach more participants because the clinics were closed due to the pandemic at the time of the study. Secondly, this was an online survey and the responses depended on judgement.

\section{Conclusion}

The current study suggest that dentistry students were closely following the pandemic measures, and were highly adapted to the preventive behaviors about COVID-19. On the other hand, the results revealed that some proportion of students showed confusions at the level of knowledge about the risk of cross-infection in dentistry procedures.

\section{Ethics}

Ethics Committee Approval: The questionnaire forms were delivered to the participants after the approval of the Gülhane Scientific Research Ethics Committee of the University of Health Sciences Turkey, on 24.09.2020 (no. 2020-370).

Informed Consent: Informed consent was obtained from all participants before the questionnaire was administered.

Peer-review: Externally peer-reviewed.

\section{Authorship Contributions}

Concept: N.O., A.A.B., Design: N.O., A.A.B., H.P.Ö., Data Collection or Processing: A.A.B., İ.H.A., Analysis or Interpretation: N.O., A.A.B., H.P.Ö., I.H.A., Literature Search: A.A.B., İ.H.A., Writing: N.O., A.A.B., H.P.Ö., I.H.A.

Conflict of Interest: No conflict of interest was declared by the authors.

Financial Disclosure: The authors declared that this study received no financial support.

\section{References}

1. Lu R, Zhao X, Li J, et al. Genomic characterisation and epidemiology of 2019 novel coronavirus: implications for virus origins and receptor binding. Lancet. 2020;395:565574.

2. Huang $C$, Wang $Y$, Li X, et al. Clinical features of patients infected with 2019 novel coronavirus in Wuhan, China. Lancet. 2020;395:497-506.

3. Wang L, Wang Y, Ye D, Liu Q. Erratum to "A review of the 2019 Novel Coronavirus (COVID-19) based on current evidence" [International Journal of Antimicrobial Agents 55/6 (2020) 105948]. Int J Antimicrob Agents. 2020;56:106137.

4. Peng X, Xu X, Li Y, Cheng L, Zhou X, Ren B. Transmission routes of 2019-nCoV and controls in dental practice. Int J Oral Sci. 2020;12:9.
5. Republic of Turkey Ministry of Health, Turkey Covid-19 Patient Table. 2020. Available from: covid19.saglik.gov.tr

6. Liu Y, Gayle AA, Wilder-Smith A, Rocklöv J. The reproductive number of COVID-19 is higher compared to SARS coronavirus. J Travel Med. 2020;27:taaa021.

7. Del Rio C, Malani PN. 2019 Novel Coronavirus-Important Information for Clinicians. JAMA. 2020;323:1039-1040.

8. Chen N, Zhou M, Dong X, et al. Epidemiological and clinical characteristics of 99 cases of 2019 novel coronavirus pneumonia in Wuhan, China: a descriptive study. Lancet. 2020;395:507-513.

9. Li Q, Guan X, Wu P, et al. Early Transmission Dynamics in Wuhan, China, of Novel Coronavirus-Infected Pneumonia. N Engl J Med. 2020;382:1199-1207.

10. Shen K, Yang Y, Wang T, et al. Diagnosis, treatment, and prevention of 2019 novel coronavirus infection in children: experts' consensus statement. World J Pediatr. 2020;16:223-231.

11. Van Doremalen N, Bushmaker T, Morris DH, et al. Aerosol and Surface Stability of SARS-CoV-2 as Compared with SARS-CoV-1. N Engl J Med. 2020;382:1564-1567.

12. Al-Amad SH, Awad MA, Edher FM, Shahramian K, Omran TA. The effect of rubber dam on atmospheric bacterial aerosols during restorative dentistry. J Infect Public Health. 2017;10:195-200.

13. Taghrir MH, Borazjani R, Shiraly R. COVID-19 and Iranian Medical Students; A Survey on Their Related-Knowledge, Preventive Behaviors and Risk Perception. Arch Iran Med. 2020;23:249-254.

14. Saqlain M, Munir MM, Rehman SU, et al. Knowledge, attitude, practice and perceived barriers among healthcare workers regarding COVID-19: a cross-sectional survey from Pakistan. J Hosp Infect. 2020;105:419-423.

15. Ataş O, Talo Yildirim T. Evaluation of knowledge, attitudes, and clinical education of dental students about COVID-19 pandemic. PeerJ. 2020;8:e9575.

16. Sarfaraz S, Shabbir J, Mudasser MA, et al. Knowledge and Attitude of Dental Practitioners Related to Disinfection during the COVID-19 Pandemic. Healthcare (Basel). 2020;8:232.

17. Harrel SK, Molinari J. Aerosols and splatter in dentistry: a brief review of the literature and infection control implications. J Am Dent Assoc. 2004;135:429-437.

18. Sun J, Xu Y, Qu Q, Luo W. Knowledge of and attitudes toward COVID-19 among parents of child dental patients during the outbreak. Braz Oral Res. 2020;34:e066.

19. Falahchai $M$, Hemmati $Y B$, Hasanzade M. Dental care management during the COVID-19 outbreak. Spec Care Dentist. 2020;1-10. 
20. Oral N, Aslan Balcı A, Peker Öztürk H, Avsever İH. Determining the knowledge, attitude and the behavior of people living in different regions of turkey in terms of dental procedures during covid-19 pandemic. ESTÜDAM Halk Sağlığı Dergisi. 2020;5:472-481.

21. Wang C, Miao L, Wang Z, Xiong Y, Jiao Y, Liu H. Emergency Management in a Dental Clinic During the Coronavirus
Disease 2019 (COVID-19) Epidemic in Beijing. Int Dent J. 2021;71:32-39.

22. Ahmed MA, Jouhar R, Adnan S, Ahmed N, Ghazal T, Adanir N. Evaluation of Patient's Knowledge, Attitude, and Practice of Cross-Infection Control in Dentistry during COVID-19 Pandemic. Eur J Dent. 2020;14:S1-S6. 